DOI: 10.1111/all.14683

Drug Allergy, Insect Sting Allergy, and Anaphylaxis

\title{
Peanut-induced anaphylaxis in children and adolescents: Data from the European Anaphylaxis Registry
}

\author{
loana Maris $^{1}$ ( ) | Sabine Dölle-Bierke ${ }^{2}$ | Jean-Marie Renaudin ${ }^{3}$ | Lars Lange ${ }^{4}$ | \\ Alice Koehli ${ }^{5}$ | Thomas Spindler ${ }^{6}$ | Jonathan Hourihane,8 | Kathrin Scherer ${ }^{9}$ | \\ Katja Nemat $^{10}$ | C. Kemen ${ }^{11}$ | Irena Neustädter ${ }^{12}$ | Christian Vogelberg ${ }^{13}$ | \\ Thomas Reese $^{14}$ | Ismail Yildiz $^{15} \mid$ Zsolt Szepfalusi $^{16}{ }^{16} \mid$ Hagen Ott $^{17}$ | \\ Helen Straube $^{18}$ | Nikolaos G. Papadopoulos ${ }^{19,20}$ | Susanne Hämmerling ${ }^{21}$ | \\ Ute Staden $^{22}$ | Michael Polz ${ }^{23}$ | Tihomir Mustakov ${ }^{24}$ | Ewa Cichocka-Jarosz ${ }^{25}$ \\ Renata Cocco $^{26}$ | Alessandro Giovanni Fiocchi ${ }^{27}$ | Montserrat Fernandez-Rivas ${ }^{28}$ | \\ Margitta Worm ${ }^{2}$ @ | Network for Online Registration of Anaphylaxis (NORA) \\ ${ }^{1}$ Bon Secours Hospital Cork/Paediatrics and Child Health, University College Cork, Cork, Ireland \\ ${ }^{2}$ Division of Allergy and Immunology, Department of Dermatology, Venereology and Allergology, Charité - Universitätsmedizin Berlin, corporate member of \\ Freie Universität Berlin, Humboldt-Universität zu Berlin, and Berlin Institute of Health, Berlin, Germany \\ ${ }^{3}$ Allergy Vigilance Network, Vandoeuvre les Nancy, France \\ ${ }^{4}$ Department of Paediatrics, St. Marien-Hospital, Bonn, Germany \\ ${ }^{5}$ Division of Allergology, University Children's Hospital Zurich, Zürich, Switzerland \\ ${ }^{6}$ Department of Paediatrics, Medical Campus Hochgebirgsklinik Davos, Davos, Switzerland \\ ${ }^{7}$ Paediatrics and Child Health, Royal College of Surgeons in Ireland, Dublin, Ireland \\ ${ }^{8}$ Children's Health Ireland, Dublin, Ireland \\ ${ }^{9}$ Medical Faculty, University of Basel, Basel, Switzerland \\ ${ }^{10}$ Practice for paediatric pneumology and allergology, Kinderzentrum Dresden-Friedrichstadt, Dresden, Germany \\ ${ }^{11}$ Department of Paediatrics, Children's Hospital WILHELMSTIFT, Hamburg, Germany \\ ${ }^{12}$ Department of Paediatrics, Hallerwiese Cnopfsche Kinderklinik, Nuremberg, Germany \\ ${ }^{13}$ Department of Paediatrics, Universitätsklinikum Carl Gustav Carus, Technical University, Dresden, Germany \\ ${ }^{14}$ Department of Paediatrics, Mathias-Spital Rheine, Rheine, Germany \\ ${ }^{15}$ Department of Paediatrics, Friedrich-Ebert-Krankenhaus, Neumuenster, Germany \\ ${ }^{16}$ Division of Paediatric Pulmonology, Allergology and Endocrinology, Department of Paediatrics and Adolescent Medicine, Competence Center Paediatrics, \\ Medical University of Vienna, Vienna, Austria \\ ${ }^{17}$ Division of Paediatric Dermatology and Allergology, Epidermolysis bullosa-Centre Hannover, Children's Hospital AUF DER BULT, Hanover, Germany \\ ${ }^{18}$ Division of Allergology, Darmstädter Kinderkliniken Prinzessin Margaret, Darmstadt, Germany \\ ${ }^{19}$ Allergy Department, 2nd Paediatric Clinic, National and Kapodistrian University of Athens, Athens, Greece \\ ${ }^{20}$ Division of Infection, Immunity\& Respiratory Medicine, University of Manchester, Manchester, UK \\ ${ }^{21}$ Division of Paediatric Pulmonology and Allergology, University Children`s Hospital Heidelberg, Heidelberg, Germany \\ ${ }^{22}$ Paediatric Pneumology \& Allergology, Medical practice Klettke/Staden, Berlin, Germany \\ ${ }^{23}$ Department of Paediatrics, GPR Klinikum, Rüsselsheim, Germany \\ ${ }^{24}$ Chair of Allergy, University Hospital Alexandrovska, Sofia, Bulgaria
}

${ }^{\dagger}$ Participating centres can be found under www.anaphylaxie.net 


\footnotetext{
${ }^{25}$ Department of Paediatrics, Jagiellonian University Medical College, Krakow, Poland

${ }^{26}$ Division of Allergy, Clinical Immunology and Rheumatology, Department of Paediatrics, Federal University of São Paulo, São Paulo, Brazil

${ }^{27}$ Paediatric Hospital Bambino Gesù IRCCS, Rome, Holy See

${ }^{28}$ Allergy Department, Hospital Clinico San Carlos, Universidad Complutense, IdISSC, ARADyAL, Madrid, Spain
}

\section{Correspondence}

Margitta Worm, Division of Allergy and Immunology, Department of Dermatology, Venerology and Allergology, Charité Universitätsmedizin Berlin, Charitéplatz 1, 10117 Berlin, Germany.

Email: margitta.worm@charite.de

\section{Funding information}

The European Anaphylaxis Registry was supported by the Network for Online Registration of Anaphylaxis NORA e. V. $\mathrm{IM}$ and $\mathrm{JH}$ received grant funding related to this work from the National Children's Research Centre, Dublin, Ireland. This work was in part supported by a grant to Margitta Worm within the KFO339. Open Access funding enabled and organized by ProjektDEAL. WOA Institution: Charite Universitatsmedizin Berlin Blended DEAL: ProjektDEAL

\begin{abstract}
Background: Peanut allergy has a rising prevalence in high-income countries, affecting $0.5 \%-1.4 \%$ of children. This study aimed to better understand peanut anaphylaxis in comparison to anaphylaxis to other food triggers in European children and adolescents. Methods: Data was sourced from the European Anaphylaxis Registry via an online questionnaire, after in-depth review of food-induced anaphylaxis cases in a tertiary paediatric allergy centre.

Results: 3514 cases of food anaphylaxis were reported between July 2007 - March 2018, 56\% in patients younger than 18 years. Peanut anaphylaxis was recorded in 459 children and adolescents ( $85 \%$ of all peanut anaphylaxis cases). Previous reactions ( $42 \%$ vs. $38 \% ; p=.001$ ), asthma comorbidity ( $47 \%$ vs. $35 \% ; p<.001$ ), relevant cofactors ( $29 \%$ vs. $22 \% ; p=.004$ ) and biphasic reactions ( $10 \%$ vs. $4 \% ; p=.001)$ were more commonly reported in peanut anaphylaxis. Most cases were labelled as severe anaphylaxis (Ring\&Messmer grade III $65 \%$ vs. $56 \%$ and grade IV $1.1 \%$ vs. $0.9 \% ; p=.001$ ). Self-administration of intramuscular adrenaline was low (17\% vs. $15 \%$ ), professional adrenaline administration was higher in non-peanut food anaphylaxis (34\% vs. $26 \%$; $p=.003$ ). Hospitalization was higher for peanut anaphylaxis (67\% vs. $54 \% ; p=.004$ ). Conclusions: The European Anaphylaxis Registry data confirmed peanut as one of the major causes of severe, potentially life-threatening allergic reactions in European children, with some characteristic features e.g., presence of asthma comorbidity and increased rate of biphasic reactions. Usage of intramuscular adrenaline as first-line treatment is low and needs to be improved. The Registry, designed as the largest database on anaphylaxis, allows continuous assessment of this condition.
\end{abstract}

\section{KEYWORDS}

anaphylaxis, food allergy, paediatrics

\section{1 | INTRODUCTION}

The population-level burden of childhood food allergy (FA) is increasing, with growing epidemic and severity of reactions. Over the last decade, several review articles have reported a significant increase in food-induced anaphylaxis related Emergency Department visits in children. ${ }^{1,2}$

Peanut is one of the major food allergens in children, with increasing prevalence. In the United States, the prevalence of peanut allergy more than tripled between 1997 and 2008, with a recent study finding another $21 \%$ increase since $2010 .^{3}$ In the UK, reported rates of peanut allergy in 3 - to 5 -year-old children increased from $0.5 \%$ in 1989 , to $1.2 \%$ in 2001-2002 in the same geographical area. ${ }^{4}$

Peanut allergy affects $1.4 \%-4.5 \%$ of children and nearly $50 \%$ of peanut allergic individuals have had a past severe reaction. ${ }^{5}$ The
European Anaphylaxis Registry reports peanut as an elicitor for anaphylaxis from infancy to young adulthood, triggering nearly one third of food-induced anaphylaxis in the paediatric cohort. ${ }^{6}$

Reactions are unpredictable in relation to occurrence, severity and outcome and occur despite the appropriate allergen avoidance. Uncertainty results in a perception of risk that adversely affects health-related quality of life (HRQoL). ${ }^{7}$ Also absence of evidence regarding reliable severity predictor markers contributes to patient's and parents' lack of control over their environment with further consequences on HRQoL.

This study aimed to provide a comparison between anaphylaxis to peanut and other food triggers in European children and adolescents, with regards to atopic history, previous reactions, cofactors, symptoms timing and severity, emergency and long-term management. 


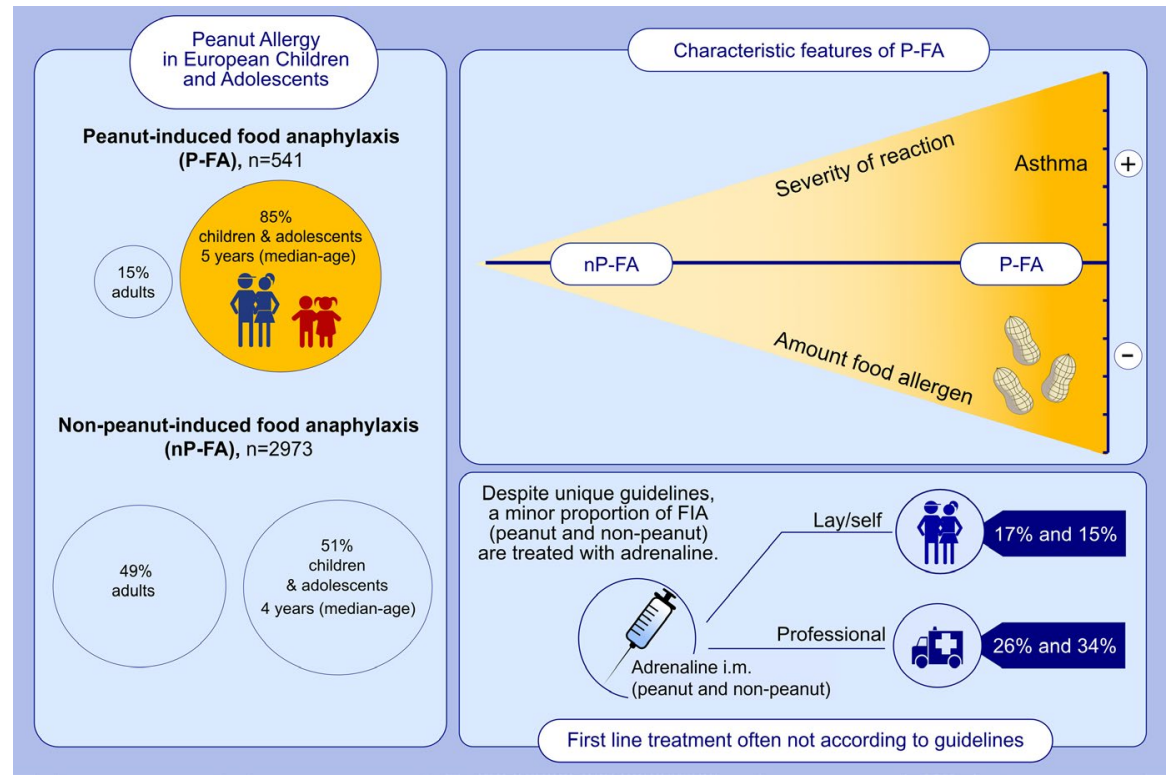

\section{GRAPHICAL ABSTRACT}

The European Anaphylaxis Registry data confirmed peanut as one of the major causes of severe allergic reactions in European children. Asthma was a more common comorbidity in peanut allergic children.Peanut anaphylaxis was more likely to be elicited by smaller amounts, and presented with more severe symptoms.The frequency of biphasic reactions was higher compared to anaphylaxis triggered by other food. Abbreviations: FIA, food-induced anaphylaxis; nP-FA, non-peanut-induced food anaphylaxis; P-FA, peanut-induced food anaphylaxis

\section{2 | MATERIAL AND METHODS}

\subsection{Data source}

Data was sourced from the European Anaphylaxis Registry, a database that collects data regarding anaphylactic reactions from 137 specialized tertiary allergy centres in ten European countries (Germany, Austria, Switzerland, Poland, Italy, Spain, Ireland, Greece, France, Bulgaria), and Brazil. Data collection is based on an online questionnaire which continuously developed over time to include additional information, mainly focused on elicitor(s), symptoms, course of reaction, cofactors, emergency treatment, diagnostic procedures and preventive/long-term management (current version 7.0). For example, the question regarding biphasic course was introduced in version 4.0 in 2010. Detailed questions on food, previous diagnosis, and the questions regarding secondline treatment were introduced in version 5.0 in 2011. The items hospitalization and intensive care unit admission were introduced in version 6.0 in 2013.

Pseudo-anonymized data of patients with anaphylaxis were reported, after in-depth review of the anaphylaxis cases by trained health care professionals from a tertiary allergy centre. On their first visit in the centre parents were asked to provide written informed consent to allow registration of the child's medical history and reaction details in the database after completion of the diagnostic workup.

Ethical approval was obtained from the Ethics Committee, Charité - Universitätsmedizin Berlin, Germany (the coordinating centre), as well as from the local Ethic Committees in all participating centres.

\section{2 | Study group}

The study cohort included patients younger than 18 years, reported between July 2007 and March 2018, with moderate and severe anaphylaxis triggered by food (Figure 1). For comparison analysis was performed on the peanut anaphylaxis subgroup versus the subgroup of anaphylaxis triggered by other food.

Only cases fulfilling the modified National Institute of Allergy and Infectious Diseases/Food Allergy and Anaphylaxis Network (NIAID/FAAN) diagnostic criteria for anaphylaxis were included. The severity of reaction was graded according to the Ring\&Messmer classification (grade II-IV). Peanut as an elicitor was ascertained by the local allergy specialist based on in-depth diagnosis.

\section{3 | Variables}

The study analysed data regarding atopic history, previous reactions, cofactors, symptoms, severity and timing of reaction, emergency treatment and long-term management. Variables were reported as a fraction of valid answer options, with multiple selections possible for some questions. All variables except for age at reaction, gender and symptoms were allowed to remain missing by item, therefore the denominator used to calculate percentages did 

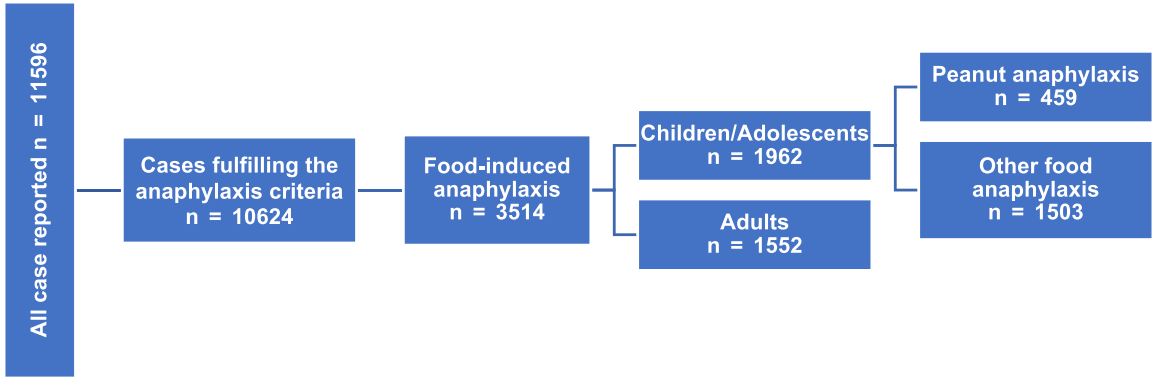

FIGURE 1 Flowchart of case selection

slightly vary from the total for some variables. Specific areas covered and the online version can be accessed through www.anaph ylaxie.net.

\subsection{Statistics}

Statistical analysis was performed using IBM SPSS Statistics 25.

Basic frequency distributions were used to describe different characteristics: demographic data, atopic history, cofactors, symptoms, severity and timing of reactions, pre-hospital and medically administered care, long-term management. Mann-Whitney $U$ test was used for comparison between the 2 subgroups; a $p$ value $<.05$ was considered to be statistically significant.

\section{3 | RESULTS}

3514 cases of food anaphylaxis were reported between July 2007 and March 2018, 56\% in patients younger than 18 years $(n=1962)$. Anaphylaxis due to peanut was recorded in 459 children and adolescents, representing $85 \%$ of all registered cases of peanut anaphylaxis ( $n=541$ cases of peanut anaphylaxis in children/adolescents and adults). Other food caused anaphylaxis in 1503 cases, with cow's milk, hen's egg, hazelnut and cashew as lead elicitors (Supplement Table S1).

Median age of affected patients was higher in the peanut subgroup compared to the other food cohort (5 years, range 8 months to 17 years vs. 4 years, range 1 month to 17 years; $p<.001$ ), with a slight predominance in boys in both subgroups (F:M ratio of 1:1.5 and 1:1.8 respectively). More than half of the peanut cohort were pre-schoolers aged $0-6$ years $(n=282 ; 61 \%)$, followed by children $7-12$ years $(n=115 ; 25 \%)$ and adolescents 13-17 years ( $n=62 ; 14 \%$ ), which was comparable to the other food cohort (Table 1).

The countries with the most frequent reports of peanut induced anaphylaxis in children/adolescents compared to the total case reports aged $<18$ years were France $(n=92 ; 23 \%)$, Germany $(n=242$; $18 \%$ ), Ireland ( $n=28 ; 17 \%)$, Switzerland $(n=65 ; 15 \%)$ and Austria ( $n=13 ; 14 \%$ ). Less than 10 cases of peanut anaphylaxis were reported from Greece, Spain, and Brazil, but also from Poland and
Bulgaria. No case of peanut induced anaphylaxis was registered in Italy (Figure 2).

\subsection{History and cofactors}

History of at least one previous reaction to peanut, usually milder, was more common ( $n=192 ; 42 \%$ ) than the same history in the other food anaphylaxis subgroup $(n=569 ; 38 \%)(p=.001)$. Previous anaphylaxis to peanut was reported in 23 cases, and to other food in 88 cases (Table 1).

Peanut allergy was already diagnosed before the recorded anaphylaxis in $45 \%$ of cases ( $n=167$, specified in $n=371$ out of 459 ), significantly higher than for other food triggers (33\%; $n=409$, specified in 1243 out of 1503) ( $p$ <.001) (Table 1).

Allergic comorbidities were frequent in both subgroups. Asthma was more common a co-disease in the peanut cohort ( $n=150$ vs. $n=359 ; p<.001)$. There was no difference in the frequency of respiratory symptoms with anaphylaxis between peanut allergic children with or without concomitant asthma (data not shown). Eczema was more frequent in the other food triggers subgroup ( $n=126$ vs. $n=525 ; p=.035$ ), and there was no statistically significant difference between the two subgroups regarding frequency of allergic rhinitis, and other food allergy (Table 1).

Relevant cofactors, potentially influencing allergenic threshold, were more commonly reported in peanut anaphylaxis ( $n=114 ; 29 \%$ ) compared to other food triggers ( $n=281 ; 22 \%)(p=.004)$. Physical exercise ( $80 \%$ vs. $77 \% ; p=.002)$ and infection were the most frequent cofactors (Table 1 ).

\section{2 | Location}

There was no statistically significant difference in terms of location for peanut or other food-induced anaphylaxis. Most events happened in the country of residence ( $97 \%$ vs. $98 \%$ ) predominantly at home $(42 \%$ vs. $50 \%)$, in school or kindergarten ( $12 \%$ vs. $9 \%$ ), and in a relative's or friend's house (11\% vs. $7 \%$ ).

Non-prepacked products were responsible for only $38 \%(n=123)$ of the peanut reactions (specified in $n=320$ out of 459), compared to other foods, where they triggered the majority of the events (63\%; $n=610$ out of 972 cases with this information available) ( $p=.005)$ 
TABLE 1 Clinical profile of peanut versus other food-induced anaphylaxis

\begin{tabular}{|c|c|c|c|c|c|}
\hline & \multicolumn{2}{|c|}{ PEANUT } & \multicolumn{2}{|c|}{ OTHER FOOD } & \multirow[b]{2}{*}{$p^{*}$} \\
\hline & $n$ & $\%$ & $n$ & $\%$ & \\
\hline Total & 459 & 100 & 1503 & 100 & \\
\hline Boys & 278 & 61 & 977 & 65 & NS \\
\hline $0-6$ years & 282 & 61 & 975 & 65 & NS \\
\hline $7-12$ years & 115 & 25 & 302 & 20 & NS \\
\hline $13-17$ years & 62 & 14 & 226 & 15 & NS \\
\hline Atopy & 296 & 64 & 991 & 66 & NS \\
\hline Asthma & 150 & $51^{f}$ & 359 & $36^{f}$ & $<.001$ \\
\hline Eczema & 126 & $43^{f}$ & 525 & $53^{f}$ & .035 \\
\hline Rhinitis & 115 & $39^{f}$ & 343 & $35^{f}$ & NS \\
\hline Food allergy & 85 & $29^{f}$ & 334 & $34^{f}$ & NS \\
\hline Previous reactions & 192 & 42 & 569 & 38 & .001 \\
\hline Milder & 126 & $66^{g}$ & 380 & $67^{g}$ & NS \\
\hline Anaphylaxis & 23 & $12^{\mathrm{g}}$ & 88 & $15^{\mathrm{g}}$ & NS \\
\hline Previous diagnosis $^{a}$ & 167 & 45 & 409 & 33 & $<.001$ \\
\hline Cofactors & 114 & 29 & 281 & 22 & .004 \\
\hline Exercise & 91 & $80^{h}$ & 215 & $77^{\mathrm{h}}$ & .002 \\
\hline Acute illness & 16 & $14^{h}$ & 36 & $13^{\mathrm{h}}$ & NS \\
\hline \multicolumn{6}{|l|}{ Symptoms } \\
\hline Skin & 418 & 91 & 1412 & $94 \%$ & .009 \\
\hline Gastrointestinal & 270 & 59 & 744 & $50 \%$ & .003 \\
\hline Respiratory & 423 & 92 & 1349 & $90 \%$ & NS \\
\hline Cardiovascular & 164 & 36 & 526 & $35 \%$ & NS \\
\hline \multicolumn{6}{|l|}{ Severity $R \& M^{e}$} \\
\hline Grade II & 155 & 34 & 644 & 43 & $<.001$ \\
\hline Grade III & 299 & 65 & 846 & 56 & $<.001$ \\
\hline Grade IV & 5 & 1.1 & 13 & 0.9 & $<.001$ \\
\hline Non-prepacked ${ }^{\text {b }}$ & 123 & 31.7 & 610 & 63 & .005 \\
\hline $\begin{array}{c}\text { Small eliciting } \\
\text { amount }^{c}\end{array}$ & 197 & 66 & 446 & 43 & $<.001$ \\
\hline Biphasic reaction $^{d}$ & 39 & 10 & 52 & 4 & .001 \\
\hline
\end{tabular}

Abbreviations: $\mathrm{n}$, number; NS, non-significant.

${ }^{a}$ Based on $n=371$ and on $n=1243$ respectively, as question was not asked from the beginning.

${ }^{b}$ Based on $n=320$ and on $n=972$ respectively, as question was not asked from the beginning.

${ }^{c}$ Based on $n=300$ and on $n=1034$ respectively, as question was not asked from the beginning.

${ }^{\mathrm{d}}$ Based on $n=404$ and on $n=1314$ respectively, as question was not asked from the beginning.

'e Severity according to Ring\&Messmer.

${ }^{f}$ Percent of cases with current concomitant atopic diseases.

${ }^{\mathrm{g}}$ Percent of cases with previous reactions.

${ }^{\text {h}}$ Percent of cases with cofactor involvement.

*Mann-Whitney $U$ test.

(Table 1). Milk and egg (63\%; $p<.001)$, but also some tree nuts $(53 \%$; $p<.001$ ) were main ingredients in non-prepacked food. Products were catered or bought (e.g., bakery, supermarket). Peanut was more often

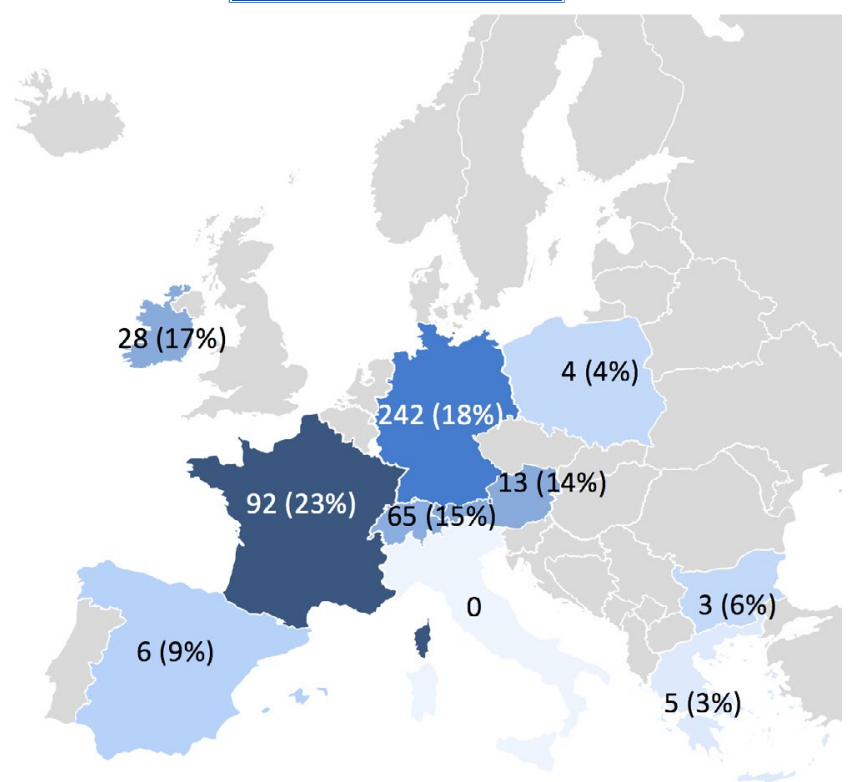

FIGURE 2 Frequency of reported peanut anaphylaxis in children and adolescents from the European Anaphylaxis Registry

listed as an ingredient or on precautionary allergen labelling compared to other food allergens ( $54 \%$ vs. $40 \% ; p=.001)$, but $45 \%(n=120)$ and $58 \%(n=386)$ respectively answered 'don't know' to this question.

Small amounts (less or equal to 1 teaspoon) were more likely to elicit the reaction in peanut anaphylaxis $(66 \% ; n=197$ specified in $n=300$ out of $459 \%$ vs. $43 \% ; n=446$ specified in $n=1034$ out of 1503; $p<.001$ ) (Figure 3)

\section{3 | Symptoms, severity and timing of reaction}

Respiratory (92\% vs. $90 \%)$ and skin (91\% vs. $94 \% ; p=.009)$ were the organ systems most frequently affected in both subgroups. Gastrointestinal symptoms were more common in peanut anaphylaxis ( $59 \%$ vs. $50 \% ; p=.003$ ) and there was no statistically significant difference for cardiovascular involvement between subgroups $(36 \%$ vs. $35 \%)$.

According to the Ring\&Messmer classification the majority of cases in both subgroups were labelled as severe anaphylaxis, however significantly more in the peanut cohort ( $p=.001$; Table 1 ).

Death was recorded in $0.7 \%(n=3)$ in the peanut cohort, and in $0.3 \%(n=5)$ in the other food cohort. The 3 cases of fatal peanut anaphylaxis occurred in 2006, 2012 and 2013. They were all female teenagers, two of them had other food allergies/atopic disease, and stress was given as possible cofactor in one case. First-line treatment was unknown in one case and provided by an emergency professional in the other two, but only one received adrenaline (im and iv) as well as oxygen and iv fluid volume. Other confirmed food triggers causing fatal anaphylaxis were cow's milk (2 cases), snail, and poppy seeds, whereas kiwi or hazelnut were suspected in another case. 


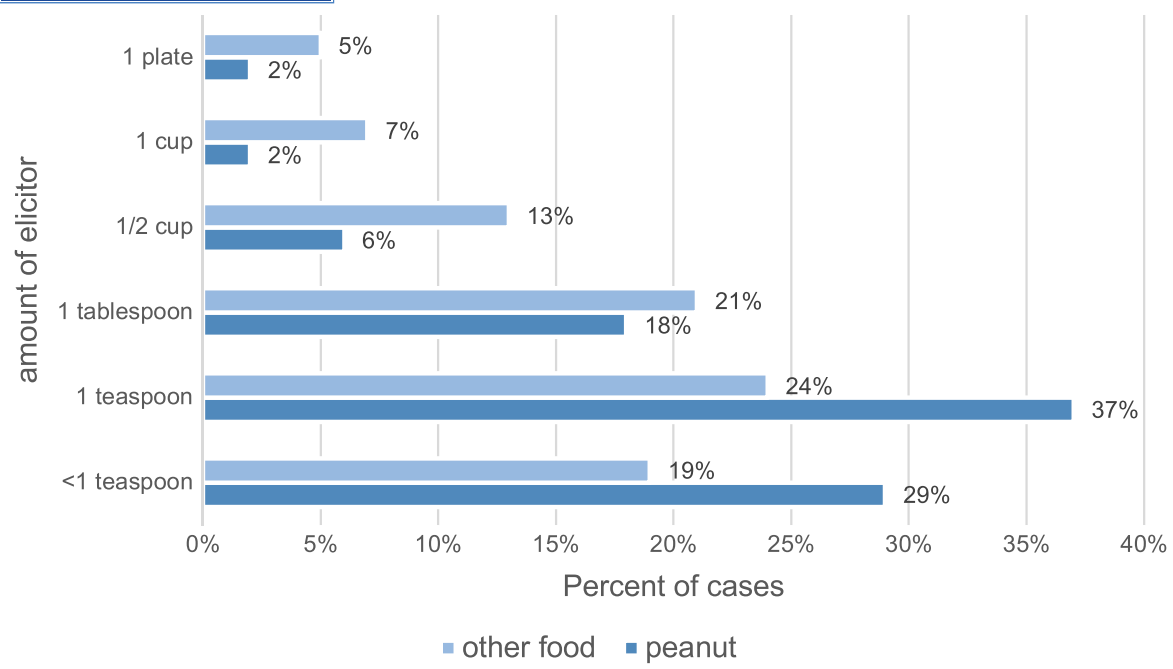

FIGURE 3 Elicitor amount triggering peanut and non-peanut induced anaphylaxis. This question was answered by $n=300$ (peanut) and $n=1034$ (non-peanut). The differences were statistical significant for each $(p<.001$, Mann-Whitney $U$ test)

Analysis of the interval between exposure and onset of symptoms revealed no differences between peanut and other food triggered anaphylaxis: $49 \%(n=226$; specified for 366 out of 459 cases) and $48 \%$ ( $n=714$; specified for 1219 out of 1503) respectively reacted within $10 \mathrm{~min}$ after exposure to the trigger, and another $18 \%(n=83)$ and $21 \%$ respectively $(n=310)$ within $30 \mathrm{~min}$. In both subgroups $7 \%$ of the patients reported a delayed reaction with an interval of more than $1 \mathrm{~h}$ (Supplement Figure S1).

The frequency of biphasic reactions, which were defined as subsequent recurrence of the same or appearance of new symptoms after initial improvement, was significantly higher for peanut anaphylaxis compared to other food triggers (10\%, specified for $n=404$ out of 459 , vs. $4 \%$, specified for $n=1314$ out of 1503 ; $p=.001$ ) (Table 1). The second reaction occurred after more than $12 \mathrm{~h}$ in $21 \%$ of the cases in both subgroups. For peanut anaphylaxis, there were no statistically significant differences between the biphasic and non-biphasic reactions in terms of age, reaction severity, and administration of adrenaline.

\subsection{Emergency treatment}

First-line treatment was administered in $89 \%$ of peanut anaphylaxis and $91 \%$ of reactions triggered by other food.

$27 \%$ in both food subgroups were solely lay treated, mainly by a family member ( $80 \%$ vs. $81 \%$ ). Another $12 \%$ and $13 \%$ respectively were initially cared for by a lay person followed by a professional. $17 \%$ ( $n=28$ ) of the peanut allergic children self-administered an adrenaline auto-injector (AAI), with a similar figure ( $n=78 ; 15 \%)$ for other food triggers. Lay treatment also included antihistamines $(p<.001)$, oral steroids and beta2-agonist inhalers $(p=.008)$ (Table 2$)$.

Emergency treatment was carried out solely by a healthcare professional in $>50 \%$ in both food subgroups. $40 \%$ versus $44 \%$ were treated by an emergency physician. Professional treatment included adrenaline (intramuscular (im), intravenous (iv), and inhalative) ( $p=.003$ ), antihistamines (iv and oral), corticosteroids (iv, oral, and rectal), or beta2-agonist (iv, oral, and inhalative) ( $p=.044$ ) (Table 2).

Almost one in two peanut allergic children (44\%) already prescribed an AAI did not use or carry the device. The percentage was slightly higher but not statistically significant for other food allergies (53\%) (Table 2).

Second-line treatment (i.e., additional doses or other drugs not used for initial management, with failure of first-line treatment) was reported in $24 \%$ of peanut allergic children ( $n=93$ out of 388 cases with this information available), with no significant difference compared to the other food subgroup. A second dose of adrenaline was administered in $5 \%(n=10)$ of peanut anaphylaxis and $6 \%(n=41)$ in the non-peanut food group (Table 2).

Hospitalization was required in a higher number for peanut anaphylaxis compared to other food (67\% of 280 cases with known hospitalization status vs. $54 \%$ of 998 cases, $p=.004$ ), but there was no difference regarding admission to Intensive Care Unit (Table 2).

Tryptase testing was performed outside the episode in $21 \%$ of cases in both subgroups. Elevated serum levels were only registered in a small percentage and with no statistically significant difference between the 2 subgroups ( $14 \%$ vs. $6 \%$ ).

\section{5 | Long-term management plan}

Almost all children received counselling about trigger avoidance. Emergency drugs prescription was significantly higher for peanut anaphylaxis (97\% vs. 93\%; $p=.009$ ), but training in an emergency plan was similar between the 2 subgroups.

AAls were more prescribed for peanut anaphylaxis compared to other food ( $96 \%$ vs. $89 \% ; p=.003$ ), as well as inhaled beta2-agonists 
TABLE 2 Emergency treatment and long-term management

\begin{tabular}{|c|c|c|c|c|c|}
\hline & \multicolumn{2}{|c|}{ PEANUT } & \multicolumn{2}{|c|}{ OTHER FOOD } & \multirow[b]{2}{*}{$p^{*}$} \\
\hline & $n$ & $\%$ & $n$ & $\%$ & \\
\hline Total & 459 & 100 & 1503 & 100 & \\
\hline First-line treatment & 407 & 89 & 1365 & 91 & NS \\
\hline Solely lay & 110 & 27 & 364 & 27 & NS \\
\hline Lay followed by professional & 57 & 14 & 172 & 13 & NS \\
\hline Solely professional & 240 & 59 & 829 & 61 & NS \\
\hline Emergency Physician ${ }^{\mathrm{a}}$ & 119 & 40 & 440 & 44 & NS \\
\hline $\begin{array}{l}\text { Self-administered im } \\
\text { adrenaline }^{\text {b }}\end{array}$ & 28 & 17 & 78 & 15 & NS \\
\hline $\begin{array}{l}\text { Self-administered } \\
\text { antihistamine }^{\mathrm{b}}\end{array}$ & 106 & 63 & 426 & 79 & $<.001$ \\
\hline Self-administered steroids ${ }^{b}$ & 76 & 46 & 270 & 50 & NS \\
\hline $\begin{array}{l}\text { Self-administered } \\
\text { beta-2-agonists }^{\mathrm{b}}\end{array}$ & 58 & 35 & 134 & 25 & .008 \\
\hline Professional adrenaline $^{c}$ & 76 & 26 & 345 & 34 & .003 \\
\hline Professional antihistamine ${ }^{c}$ & 201 & 68 & 656 & 66 & NS \\
\hline Professional steroids ${ }^{c}$ & 220 & 74 & 704 & 70 & NS \\
\hline Professional beta-2-agonists ${ }^{c}$ & 91 & 31 & 247 & 25 & .044 \\
\hline Failure to use $A A I^{d}$ & 22 & 44 & 89 & 53 & NS \\
\hline Second-line treatment ${ }^{\mathrm{e}}$ & 93 & 24 & 248 & 19 & NS \\
\hline Second dose adrenaline & 10 & 5 & 41 & 6 & NS \\
\hline Admission $^{f}$ & 170 & 67 & 493 & 54 & .004 \\
\hline $\mathrm{ICU}$ & 16 & 6 & 49 & 5 & NS \\
\hline \multicolumn{6}{|l|}{ Prophylaxis } \\
\hline $\begin{array}{l}\text { Prescription of emergency } \\
\text { drugs }\end{array}$ & 446 & 97 & 1398 & 93 & .009 \\
\hline AAI & 426 & 93 & 1246 & 89 & .003 \\
\hline
\end{tabular}

Abbreviations: $n$, number; NS, non-significant.

${ }^{a}$ Based on $n=297$ and $n=1001$ respectively, who received professional treatment.

${ }^{b}$ Based on $n=167$ and $n=536$ respectively, who received lay treatment.

${ }^{\mathrm{C}}$ Aggregated adrenaline (im, iv, inhaled), aggregated antihistamines (iv, oral), aggregated steroids (iv, oral, rectal), aggregated beta-2-agonists (iv, oral, inhaled) based on $n=297$ and $n=1001$ respectively, who received professional treatment.

${ }^{\mathrm{d} B a s e d}$ on $n=50$ and $n=167$ respectively, who had AAl prescribed.

${ }^{\text {e}}$ Based on $n=388$ and $n=1279$ respectively, as question was not asked from the beginning.

${ }^{f}$ Based on $n=254$ and $n=920$ respectively, as question was not asked from the beginning.

${ }^{g}$ Based on 238 and $n=910$ respectively, as question was not asked from the beginning.

*Mann-Whitney $U$ test.
(45\% vs. $36 \% ; p=.002$ ), with no difference for antihistamines and corticosteroids (Table 2).

\section{4 | DISCUSSION}

Food is the leading known cause of anaphylactic reactions for children and adolescents in emergency departments in the United States and Europe, with geographical variations according to local dietary habits and food exposures. ${ }^{8}$ Peanut is not only one of the most common food trigger, but also one of the commonest trigger in anaphylaxis fatalities in the UK and USA. ${ }^{7}$ The authors chose to provide a comparison between anaphylaxis to peanut and other food triggers, although the different foods included and analysed as a group represent different entities and considerably different diseases.

Within the European Anaphylaxis Registry, Western European countries reported the majority of peanut induced anaphylaxis cases, but the Registry doesn't collect data from all over Europe. There was no overlap between these countries and the ones with the highest reports of food triggered anaphylaxis, in particular Mediterranean countries like Greece, Spain and Italy with high frequency of food allergy but not peanut. Italy interestingly had no reports of peanut anaphylaxis in children and adolescents, this 
could be explained by the fact that although Italy has 2 paediatric and 3 adult participating centres, the main reporting centre is an adult unit.

The findings of the European Anaphylaxis Registry support previously published findings from other multi (MIRABEL) ${ }^{9}$ - or single (Riley)-centred registries ${ }^{10}$ that peanut allergy is more common in pre-school aged boys. These data bases included children with peanut allergy and/or sensitization not only cases of peanut induced anaphylaxis. Similar demographics with predominance of males in younger age groups was described in several food-induced anaphylaxis studies. ${ }^{11-13}$ The lower median age in the other food subgroup is likely explained by cow's milk and egg being the predominant elicitors of allergic reactions in children in the first two years of life.

History of previous reactions more frequent in peanut allergic children (42\%), although with no difference for previous anaphylaxis, associated with the fact that $45 \%$ of peanut anaphylaxis cases were already diagnosed prior to the event, confirm the high risk of anaphylaxis and the unpredictability of further reactions in terms of severity in peanut allergic children. Sicherer et al. ${ }^{14}$ showed that almost $50 \%$ of children experience accidental exposure to peanut within 2 years of their first reaction, proving that avoidance is not enough. The Riley Registry ${ }^{10}$ reported anaphylaxis with second exposures in $33.9 \%$ of children, and in $33.3 \%$ of children who had anaphylaxis with first exposure. On a larger scale (38.408 children) Gupta et al. ${ }^{3}$ reports an estimated $42.3 \%$ of children with a food allergy to have a history of at least one severe reaction, and $42 \%$ to have at least one lifetime food allergy-related visit to the emergency department.

The majority of cases had a background of atopy, regardless of the eliciting food, with similar frequencies for atopic dermatitis, allergic rhinitis, asthma, and other food allergies/sensitizations to other studies. ${ }^{9}$ Eczema was more frequent in children allergic to foods other than peanut, likely in the younger group of infants and toddlers with cow's milk and/or egg allergy, in line with the natural history of this condition. Asthma was more common in the peanut anaphylaxis cohort. Leickly et al ${ }^{10}$ reports that children with peanut anaphylaxis were significantly more likely to have asthma $(p<.001)$ and other food allergies $(p=.04)$ than those with non-anaphylactic reactions to peanut.

The prevalence of cofactors in anaphylaxis is reported to be around $30 \%$ in adults ${ }^{15}$ and $18 \%$ in children. ${ }^{16}$ Our data reports relevant cofactors more frequently in peanut anaphylaxis $(29 \%)$ compared to other food triggers (22\%), with physical exercise in the lead. Previous data from the Registry ${ }^{6,16}$ also reported physical exercise to be the most frequent cofactor, followed by medication. There is a lack of published data on other large children anaphylaxis cohorts for comparison.

Non-prepacked food products were only responsible for one out of three peanut reactions, but for the majority of the events triggered by other food. It is likely that other food triggers e.g., egg, milk are more common ingredients in non-prepacked food compared to peanut. However peanut was more likely to be listed as an ingredient, and the lower percentage of unknown data regarding labelling in the peanut cohort along with the lower consumption of non-prepacked food are hopefully indicative of a higher compliance to allergen avoidance in this group. A study on peanut-containing foods with precautionary labelling detected low levels of peanut in only two out of 38 products. ${ }^{17}$ Another study ${ }^{18}$ reported detectable amounts of three allergens (peanut, milk and egg) in 5.3\% of products with precautionary labelling and in $1.9 \%$ of products without. These issues still have to be addressed. ${ }^{19}$ Small amounts of allergen were more likely to trigger peanut anaphylaxis (66\%) highlighting again the high risk of this trigger.

The interval between exposure and onset of symptoms was less than $10 \mathrm{~min}$ in the majority of cases, with no difference between peanut and other food, and with figures similar to previous analysis. ${ }^{6,20}$ Anaphylaxis appeared in a delayed pattern in a very small percentage of cases, again regardless of the food trigger.

Biphasic reactions were significantly more frequent in peanut anaphylaxis (10\%) versus other food triggers (4\%). The latter figure is in keeping with previous analysis of the children/adolescent cohort $(5 \%){ }^{6}$ and also with the NORA report on the mixed paediatric and adult population ( $4 \%$ in food-induced anaphylaxis). ${ }^{20}$ Similar $^{13}$ but also lower figures ${ }^{21}$ were reported in other multicentre studies on paediatric anaphylaxis.

A possible link is with the higher frequency of severe anaphylaxis in the peanut cohort (65\% grade III) versus the total children/adolescent cohort $(47 \%)^{6}$ or the mixed paediatric/adult cohort (38.3\%). ${ }^{20}$ However, there was no difference in reaction severity between the children who experienced a biphasic reaction and the ones who did not. Age 6 to 9 years was reported as one of five independent predictors for biphasic reactions, ${ }^{22}$ not confirmed in our data. Sometimes the prevalence of biphasic anaphylaxis is difficult to assess, depending on the time interval of patient observation post anaphylaxis, also symptoms occurring later might not be recognized as being related to the initial reaction. A systematic review and meta-analysis on predictors of biphasic analysis found food triggers to be associated with decreased risk for biphasic reactions. ${ }^{23}$

Only $36 \%$ of the peanut anaphylactic reactions and $40 \%$ of the reactions triggered by other food were initially treated by a lay person, including self-administration of im adrenaline. There was no statistically significant difference between subgroups regarding self-administration of im adrenaline, but failure to apply/carry an adrenaline auto-injector was registered in $44 \%$ of peanut anaphylaxis and in $53 \%$ of the cases allergic to other food, which matches the reports of studies evaluating real-world use of AAI. ${ }^{24}$ In a community-based survey uncertainty about the severity of the reaction, fear of side effects, and difficulties deciding which drugs to use were identified as reasons for not applying AAls. ${ }^{25}$

Professional emergency treatment was mainly carried out by an emergency physician. Only 1 in 4 children with peanut anaphylaxis treated by a healthcare professional received adrenaline as first-line treatment, despite the current guidelines reflecting strong expert opinion, that classifies im adrenaline as first-line treatment of anaphylaxis. ${ }^{26}$ Surprisingly, professional administration of adrenaline (im, iv, inhaled) was significantly higher in food anaphylaxis other than peanut compared to peanut anaphylaxis. 
Despite recommendations, second- and third line drugs like antihistamines, steroids and beta 2 -agonists were used as first-line drugs in the majority of the reactions, similar to other studies.

This supports a previous assertion that adrenaline is under-used in anaphylaxis treatment. A similar or lower proportion was reported for children and/or adults in other populations. Data from several cohort studies show the extent of under-treatment of anaphylaxis and the low rate of adrenaline use. ${ }^{26}$ US studies report higher usage of adrenaline in emergency setting, compared to Europe, with the majority of paediatric emergency medicine physicians (93.5\%) correctly identifying adrenaline as first-line treatment in anaphylaxis, yet only $66.9 \%$ choosing the im route. ${ }^{27}$

Aiming to evaluate the variation in the Emergency Department (ED) care of children with anaphylaxis, Michelson et al, $2016{ }^{28}$ performed a retrospective cross-sectional study on data from 35 hospitals, on ED visits with a primary diagnosis of anaphylaxis over a period of 4 years, in children aged 1 month to 18 years. The least variation regarding adjunct therapies was observed in the use of $\mathrm{H} 1$-antihistamines and steroids, which were also the most frequent administered drugs. The study did not assess the frequency of adrenaline administration because it was assumed it is commonly given prior to hospital arrival.

Second-line treatment was required in less than $25 \%$ of professionally treated children (peanut cohort $24 \%$ vs. other food cohort $19 \%$ ), and a second dose of adrenaline was administered in $5 \%$ vs. $6 \%$ (peanut vs. other food). Recent data from the Registry showed that $3.7 \%$ of professionally managed anaphylaxis received a second dose of adrenaline, ${ }^{26}$ with higher figures in a mixed but smaller paediatric/ adult cohort (13\%; $18.5 \%$ for children only). ${ }^{29}$

Hospitalization was higher in the peanut cohort, likely due to the difference in anaphylaxis severity (grade III 65\% vs. 56\%; $p=.001$ ). Despite the fact that most cases were labelled as severe anaphylaxis, only $6 \%$ required admission to the Intensive Care Unit, and $0.7 \%$ had a fatal outcome.

\subsection{Strength and limitations}

A large cohort of 459 children and adolescents with severe peanut allergy, from different European countries, allowed for a comprehensive view of the clinical characteristics of peanut triggered anaphylaxis.

The main limitation of our analysis is its retrospective nature resulting in missing data, also due to different time points of implementation of the different questions asked.

Overall this data confirmed peanut as one of the major causes of severe, potentially life-threatening allergic reactions in children in Europe. Peanut anaphylaxis shows some characteristic features e.g., the presence of asthma comorbidity and the increased rate of biphasic reactions, conditions which may even be linked to each other. Clinicians should be aware of such characteristic findings for peanut allergy and consider these in the management of peanut anaphylactic patients.

\section{ACKNOWLEDGEMENTS}

We thank all patients and/or their parents for their support of providing data on the occurrence of anaphylaxis to the Anaphylaxis Registry. We would like to thank the study personnel of every single centre for patient counselling and data entry: J. Grünhagen, M. Wittenberg, K. Beyer, A. Henschel, S. Küper (Berlin, Germany), A. Möser (Jena, Germany), T. Fuchs (Göttingen, Germany), F. Ruëff (Munich, Germany), B. Wedi, G. Hansen, T. Buck, J. Büsselberg, R. Drägerdt, L. Pfeffer (Hanover, Germany), H. Dickel, C. Körner-Rettberg (Bochum, Germany), H. Merk, S. Lehmann (Aachen, Germany), A. Bauer, A. Nordwig, S. Zeil and C. Hannapp (Dresden, Germany), N. Wagner (Erlangen, Germany), E. Rietschel, N. Hunzelmann, I. Huseynow (Cologne, Germany), R. Treudler, S. Aurich, F. Prenzel (Leipzig, Germany), L. Klimek, O. Pfaar (Wiesbaden, Germany), N. Reider (Innsbruck, Austria), W. Aberer, E. Varga (Graz, Austria), B. Bogatu, P. Schmid-Grendelmeier, R. Guggenheim (Zurich, Switzerland), F. Riffelmann (Schmallenberg, Germany), B. Kreft (Halle, Germany), K. Kinaciyan, L. Hartl, C. Ebner, F. Horak (Vienna, Austria), R. Brehler (Münster, Germany), J. Witte, M. Buss, S. Hompes (Hamburg, Germany), T. Bieber, S. Gernert, M. Bücheler (Bonn, Germany), U. Rabe (Treuenbritzen, Germany), W. Brosi (Würzburg, Germany), S. Nestoris (Lippe-Lemgo, Germany), T. Hawranek, R. Lang (Salzburg, Austria), R. Bruns (Greifswald, Germany), C. Pföhler (Homburg, Germany), P. Eng (Aarau and Lucerne, Switzerland), S. Schweitzer-Krantz, S. Meller (Düsseldorf, Germany), H. Rebmann, J. Fischer (Tübingen, Germany), G. Stichtenoth (Lübeck, Germany), S. Thies (Schwedt, Germany), M. Gerstlauer (Augsburg, Germany), P. Utz (Wangen im Allgäu, Germany), formerly I. Neustädter now J. Klinge (Fürth, Germany), S. Volkmuth (Velbert, Germany), S. Plank-Habibi (Alzenau, Germany), B. Schilling (Passau, Germany), A. Kleinheinz, A. Brückner (Buxtehude, Germany), K. Schäkel (Heidelberg, Germany), I. Manolaraki (Athen, Greece), M. Kowalski (Lodz, Poland), K. Solarewicz-Madajek (Wroclaw, Poland), S. Tscheiller and all members of the Allergy vigilance network, France, J. Seidenberg (Oldenburg, Germany), V. Cardona (Barcelona, Spain), B. Garcia (Pamplona, Spain), M. Bilo (Ancona, Italy), N. Cabañes Higuero (Toledo, Spain), A. Vega Castro (Guadalajara, Spain), I. Poziomkowska-Gęsicka (Szczecin, Poland), S. Büsing (Osnabrück, Germany), C. Virchow (Rostock, Germany), G. Christoff (Sofia, Bulgaria), U. Jappe (Borstel, Germany), S. Müller (Freiburg, Germany), F. Knöpfel (Norderney, Germany), A-K. Correard (Cork, Ireland), B. Rogala (Silesia, Poland), A. Montoro (Madrid, Spain), A. Brandes (Frankfurt/Oder, Germany), A. Muraro (Padua, Italy), N. Zimmermann (Potsdam, Germany), D. Hernandez (Valencia, Spain), P. Minale (Genua, Italy), J. Niederwimmer und B. Zahel (Linz, Austria), L. Dahdah and S. Arasi (Rome, Italy), A. Reissig (Gera, Germany), F. Eitelberger (Wels, Austria), R. Asero (Milan, Italy), F. Hermann, S. Zeidler (St. Augustin, Germany), S. Pistauer (Sylt/Westerland, Germany), M. Geißler (Ribnitz-Damgarten, Germany), L. Ensina (Sao Paulo, Brazil), A. Plaza Martin (Barcelona, Spain), J. Meister (Aue, Germany), S. Stieglitz (Wuppertal, Germany), E. Hamelmann (Bielefeld, Germany). 


\section{CONFLICT OF INTEREST}

Dr Maris reports grants from National Children's Research Centre, Dublin, Ireland, during the conduct of the study. Dr Hourihane reports grants from National Children's Research Centre, Dublin, Ireland, during the conduct of the study; grants and personal fees from Aimmune Therapeutics, grants from DBV Technologies, grants from Johnson and Johnson, outside the submitted work, and he is President of Irish Association of Allergy and Immunology and Chair of Irish Food Allergy Network, which both receive unrestricted educational grants from industry, including manufacturers of infant formulae and adrenaline autoinjectors. Dr Scherer Hofmeier reports personal fees from Allergopharma, Sanofi-Aventis, Shire (Switzerland), Novartis outside the submitted work. Dr Neustädter reports personal fees from ALK, personal fees from Nutricia, personal fees from Allergopharma, personal fees from ThermoFisher, during the conduct of the study. Dr Papadopoulos reports personal fees from Novartis, personal fees from Nutricia, personal fees from HAL, personal fees from MENARINI/FAES FARMA, personal fees from SANOFI, personal fees from MYLAN/MEDA, personal fees from BIOMAY, personal fees from AstraZeneca, personal fees from GSK, personal fees from MSD, personal fees from ASIT BIOTECH, personal fees from Boehringer Ingelheim, grants from Gerolymatos International SA, grants from Capricare, outside the submitted work. Dr Worm reports other from Regeneron Pharmaceuticals, other from DBV Technologies S.A, other from Stallergenes $\mathrm{GmbH}$, other from HAL Allergie $\mathrm{GmbH}$, other from Bencard Allergie $\mathrm{GmbH}$, other from Allergopharma GmbH \& Co. KG , other from ALK-Abelló Arzneimittel $\mathrm{GmbH}$, other from Mylan Germany $\mathrm{GmbH}$, other from Leo Pharma $\mathrm{GmbH}$, other from Sanofi-Aventis Deutschland $\mathrm{GmbH}$, other from Aimmune Therapeutics UK Limited, other from Actelion Pharmaceuticals Deutschland $\mathrm{GmbH}$, other from Novartis AG, other from Biotest AG, other from AbbVie Deutschland GmbH \& Co. KG other from Lilly Deutschland $\mathrm{GmbH}$, outside the submitted work. The rest of the authors declare that they have no relevant conflicts of interest.

\section{ORCID}

loana Maris (iD) https://orcid.org/0000-0001-6734-2329

Zsolt Szepfalusi (D) https://orcid.org/0000-0003-4852-3102

Margitta Worm (D) https://orcid.org/0000-0002-3449-1245

\section{REFERENCES}

1. Motosue MS, Bellolio MF, Van Houten HK, Shah ND, Campbell RL. Increasing emergency department visits for anaphylaxis, 20052014. J Allergy Clin Immunol Pract. 2017;5(1):171-175.e3. https:// doi.org/10.1016/J.JAIP.2016.08.013

2. Turner PJ, Gowland MH, Sharma V, et al. Increase in anaphylaxis-related hospitalizations but no increase in fatalities: an analysis of United Kingdom national anaphylaxis data, 1992-2012. J Allergy Clin Immunol. 2015;135(4):956-963.e1. https://doi.org/10.1016/j. jaci.2014.10.021

3. Gupta RS, Warren CM, Smith BM, et al. The public health impact of parent-reported childhood food allergies in the United States. Pediatrics 2018;142(6):e20181235. https://doi.org/10.1542/ peds.2018-1235
4. Custovic A. Epidemiology of allergic diseases. Middleton's Allergy Essentials. 2017:51-72. UK: Middletons Allergy Essentials. https:// doi.org/10.1016/B978-0-323-37579-5.00003-9

5. Greenhawt $M$, Marsh $R$, Gilbert $H$, Sicherer $S$, DunnGalvin $A$, Matlock D. Understanding caregiver goals, benefits, and acceptable risks of peanut allergy therapies. Ann Allergy, Asthma Immunol. 2018;121(5):575-579. https://doi.org/10.1016/j.anai.2018.06.018

6. Grabenhenrich LB, Dölle S, Moneret-Vautrin A, et al. Anaphylaxis in children and adolescents: the European Anaphylaxis Registry. J Allergy Clin Immunol. 2016;137(4):1128-1137.e1. https://doi. org/10.1016/j.jaci.2015.11.015

7. Turner PJ, Baumert JL, Beyer K, et al. Can we identify patients at risk of life-threatening allergic reactions to food? Allergy Eur J Allergy Clin Immunol. 2016;71(9):1241-1255. https://doi.org/10.1111/all.12924

8. Lyons S, Clausen M, Knulst AC, et al. Prevalence of food sensitization and food allergy in children across Europe. J Allergy Clin Immunol Pr. 2020;8(8):2736-2746.e9.

9. Deschildre A, Elegbédé CF, Just J, et al. Peanut-allergic patients in the MIRABEL survey: characteristics, allergists' dietary advice and lessons from real life. Clin Exp Allergy. 2016;46(4):610-620. https:// doi.org/10.1111/cea.12681

10. Leickly FE, Kloepfer KM, Slaven JE, Vitalpur G. Peanut allergy: an epidemiologic analysis of a large database. J Pediatr. 2018;192:223228.e1. https://doi.org/10.1016/j.jpeds.2017.09.026

11. Rudders S, Banerji A, Clark S, Camargo CJ. Age-related differences in the clinical presentation of food-induced anaphylaxis. J Pediatr. 2011;58(2):326-328.

12. Tejedor Alonso MA, Moro-Moro M, Mugica Garcia MV. Epidemiology of anaphylaxis. Clin Exp Allergy. 2015;45:1027-1039.

13. Jeon $\mathrm{YH}$, Ahn K, et al. Infantile anaphylaxis in Korea: a multicenter retrospective case study. J Korean Med Sci. 2019;34(13):e10.

14. Sicherer SH, Furlong TJ, Muñoz-Furlong A, Burks AW, Sampson HA. A voluntary registry for peanut and tree nut allergy: characteristics of the first 5149 registrants. J Allergy Clin Immunol. 2001;108(1):128-132. https://doi.org/10.1067/mai.2001.115755

15. Wölbing F, Fischer J, Köberle M, Kaesler S, Biedermann T. About the role and underlying mechanisms of cofactors in anaphylaxis. Allergy Eur J Allergy Clin Immunol. 2013;68(9):1085-1092. https:// doi.org/10.1111/all.12193

16. Hompes S, Köhli $\mathrm{A}$, Nemat $\mathrm{K}$, et al. Provoking allergens and treatment of anaphylaxis in children and adolescents - data from the anaphylaxis registry of German-speaking countries. Pediatr Allergy Immunol. 2011;22(6):568-574. https://doi. org/10.1111/j.1399-3038.2011.01154.x

17. Robertson ON, Hourihane JO, Remington BC, Baumert JL, Taylor SL. Survey of peanut levels in selected Irish food products bearing peanut allergen advisory labels. Food Addit Contam Part A. 2013;30(9):1467-1472. https://doi.org/10.1080/19440 049.2013 .804953

18. Ford LS, Taylor SL, Pacenza R, Niemann LM, Lambrecht DM, Sicherer $\mathrm{SH}$. Food allergen advisory labeling and product contamination with egg, milk, and peanut. J Allergy Clin Immunol. 2010;126(2):384385. https://doi.org/10.1016/J.JACI.2010.05.034

19. Blom WM, Michelsen-Huisman AD, van Os-Medendorp $\mathrm{H}$, et al. Accidental food allergy reactions: products and undeclared ingredients. J Allergy Clin Immunol. 2018;142(3):865-875. https://doi. org/10.1016/j.jaci.2018.04.041

20. Worm M, Moneret-Vautrin A, Scherer K, et al. First European data from the network of severe allergic reactions (NORA). Allergy Eur J Allergy Clin Immunol. 2014;69(10):1397-1404. https://doi. org/10.1111/all.12475

21. Orhan F, Canitez Y, Bakirtas A, et al. Anaphylaxis in Turkish children: a multi-centre, retrospective, case study. Clin Exp Allergy. 2011;41(12):1767-1776.

22. Alqurashi W, Stiell I, Chan K, Neto G, Alsadoon A, Wells G. Epidemiology and clinical predictors of biphasic reactions in children 
with anaphylaxis. Ann Allergy, Asthma Immunol. 2015;115(3):217223.e2. https://doi.org/10.1016/j.anai.2015.05.013

23. Lee S, Bellolio MF, Hess EP, Erwin P, Murad MH, Campbell RL. Time of onset and predictors of biphasic anaphylactic reactions. A systematic review and meta-analysis. J Allergy Clin Immunol Pract. 2015;3(3):408-416.e2. https://doi.org/10.1016/j.jaip.2014.12.010

24. Gaziel Yablowitz M, Dölle S, Schwartz DG, Worm M. Proximitybased emergency response communities for patients with allergies who are at risk of anaphylaxis: clustering analysis and scenario-based survey study. JMIR mHealth uHealth. 2019;7(8):e13414. https://doi.org/10.2196/13414

25. Simons FER, Clark S, Camargo CA. Anaphylaxis in the community: Learning from the survivors. J Allergy Clin Immunol. 2009;124(2):301-306. https://doi.org/10.1016/j.jaci.2009.03.050

26. Grabenhenrich LB, Fernández-Rivas M, Dölle-Bierke S, Worm M. Repeated epinephrine doses: an analysis of the European Anaphylaxis Registry, 2007-2018. J Allergy Clin Immunol Pract. 2019;7(8):2935-2937. https://doi.org/10.1016/J.JAIP.2019.05.040

27. Grossman SL, Baumann BM, Garcia Peña BM, Linares MY-R, Greenberg B, Hernandez-Trujillo VP. Anaphylaxis knowledge and practice preferences of pediatric emergency medicine physicians: a national survey. J Pediatr. 2013;163(3):841-846. https://doi. org/10.1016/j.jpeds.2013.02.050
28. Michelson KA, Monuteaux MC, Neuman MI. Variation and trends in anaphylaxis care in United States Children's hospitals. Acad Emerg Med. 2016;23(5):623-627. https://doi.org/10.1111/ acem.12922

29. Manivannan V, Campbell RL, Bellolio MF, Stead LG, Li JT, Decker W. Factors associated with repeated use of epinephrine for the treatment of anaphylaxis. Ann Allergy Asthma Immunol. 2009;103(5):395-400.

\section{SUPPORTING INFORMATION}

Additional supporting information may be found online in the Supporting Information section.

How to cite this article: Maris I, Dölle-Bierke S, Renaudin JM, et al. Peanut induced anaphylaxis in children and adolescents: Data from the European Anaphylaxis Registry. Allergy.

2021;76:1517-1527. https://doi.org/10.1111/all.14683 